enlarged. Brain MRI showed haemorrhagic infarction in both thalami, extending to the internal capsule on the right and in the peripheral posterior left parietal lobe. Carotid angiography showed occlusion of the vein of Galen, inferior sagittal sinus, and straight sinus consistent with thrombosis.

Routine biochemical and haematological screens were normal. Lupus anticoagulant and cardiolipin antibodies were not detected. The international normalised ratio was 1.0 (normal range $<1 \cdot 2$ ) and the activated partial thromboplastin time (APTT) was 30 seconds (normal range 20-34 seconds). Antithrombin III and protein C and $S$ concentrations were normal. The activated protein $\mathrm{C}$ resistance ratio (a ratio of the APTT measured in the presence of added activated protein $\mathrm{C}$ to the APTT measured in its absence) was 1.27 (normal $>2.5$ ). Genetic testing disclosed homozygosity for the factor $\mathrm{V}$ mutation (using the method described by Bertina et al, ${ }^{3}$ after amplification of the factor $\mathrm{V}$ cDNA by the polymerase chain reaction, the cDNA fragment was enzymatically digested and hybridised with the bioatinylated oligonucleotide specific for the allele of interest). Anticoagulation was started with intravenous heparin and dexamethasone was given. Her conscious level deteriorated over the next 24 hours despite continued therapeutic anticoagulation. She developed fixed dilated pupils, impaired reflex ocular movements, and exhibited extensor posturing in response to painful stimuli. Anticoagulation was continued throughout, and two weeks after admission further MRI showed progression of thrombus into the lateral sinus and left jugular bulb. Over the next four weeks her conscious state improved. She began to follow commands appropriately; ocular movements returned to normal, but she remained mute with an asymmetric spastic tetraparesis, left worse than right. Over the subsequent seven months, speech returned to near normal with corresponding improvement in upper and lower limb function. She is now independent in all daily activities despite bilateral upper motor neuron signs and a mild trunkal ataxia. Progress of the cerebral venous thrombosis will be followed up by MRI and it is intended to continue anticoagulation indefinitely.

Activated protein $\mathrm{C}$ is a natural inhibitor of blood coagulation which acts by inactivating plasma cofactors $\mathrm{Va}$ and VIIIa. Activated protein $\mathrm{C}$ resistance is known to occur in up to $50 \%$ of people with a personal and family history of deep venous thrombosis ${ }^{4}$ compared with a prevalence of about $5 \%$ in the general population. The genetic defect responsible for most cases of activated protein $\mathrm{C}$ resistance is now known to be a single point mutation in the factor $\mathrm{V}$ gene on chromosome $1 .^{3}$ This codes for a defective factor $\mathrm{V}$ molecule which is not properly inactivated by activated protein C.

Women taking the oral contraceptive pill who also have activated protein $\mathrm{C}$ resistance are considered to have a higher risk of venous thrombosis than women with activated protein C resistance alone. Oestrogens in replacement doses have not been shown to increase the risk of thrombosis but were stopped in the present case. There are no clear guidelines to the duration of anticoagulation after cerebral venous thrombosis, with or without activated protein $\mathrm{C}$ resistance. The decision to continue lifelong anticoagulation in this patient was based on the catastrophic nature of the venous thrombosis and on the increased risk of future thrombosis as a result of homozygosity for the factor $\mathrm{V}$ mutation.

Of the patient's family, her mother and one brother are heterozygous for the factor $\mathrm{V}$ mutation and another brother is homozygous. They have been advised that they should receive standard prophylaxis against venous thrombosis for procedures with thrombotic risk but that full anticoagulation would only be indicated for a proved thrombosis.

TE KIMBER Departments of Medicine and Neurology
JV LLOYD J Litology Unit Haematology Unit
BP BROPHY Neurosurgery Unit PD THOMPSON

Departments of Medicine and Neurology, Royal
Adelaide Hospital, Adelaide, Australia.

Correspondence to: $\operatorname{Dr} \mathrm{T}$ E Kimber, Neurology Unit, Royal Adelaide Hospital, North Terrace, Adelaide SA 5000, Australia.

1 Svensson PJ, Dählback B. Resistance to activated protein $\mathrm{C}$ as a basis for venous thrombosis. Lancet 1994;330:517-22.

2 Bridey F, Wolff M, Laissy JP, Morin V, Lefebvre $M$, de Prost D. Fatal cerebral vefebvre $M$, de Prost D. Fatal cerebral Factor V Leiden Mutation and the use Factor V Leiden Mutation and the use
of oral contraceptives. Thrombostasis and of oral contraceptives.
Haemostasis 1995;74:1382.

3 Bertina R, Koeleman B, Koster T, et al. Mutation in blood coagulation factor $\mathrm{V}$ associated with resistance to activated protein $\mathrm{C}$. Nature 1994;369:64-7.

4 Griffin JH, Evatt B, Wideman C, Fernandez JA. Anticoagulant $C$ pathway defective in majority of thrombophilic patients. Blood 1993;82:1989-93.

5 Vandenbroucke J, Koster T, Briet E, Reitsma $P$, Bertina R, Rosendaal F. Increased risk of venous thrombosis in oral contraceptive venous thrombosis in oral contraceptive
users who are carriers of factor $\mathrm{V}$ Leiden mutation. Lancet 1994;344:1453-6.

\section{Isolated dysarthria caused by a right} paravermal infarction

Information on the topography of speech disorders in cerebellar disease is lacking. ${ }^{1}$
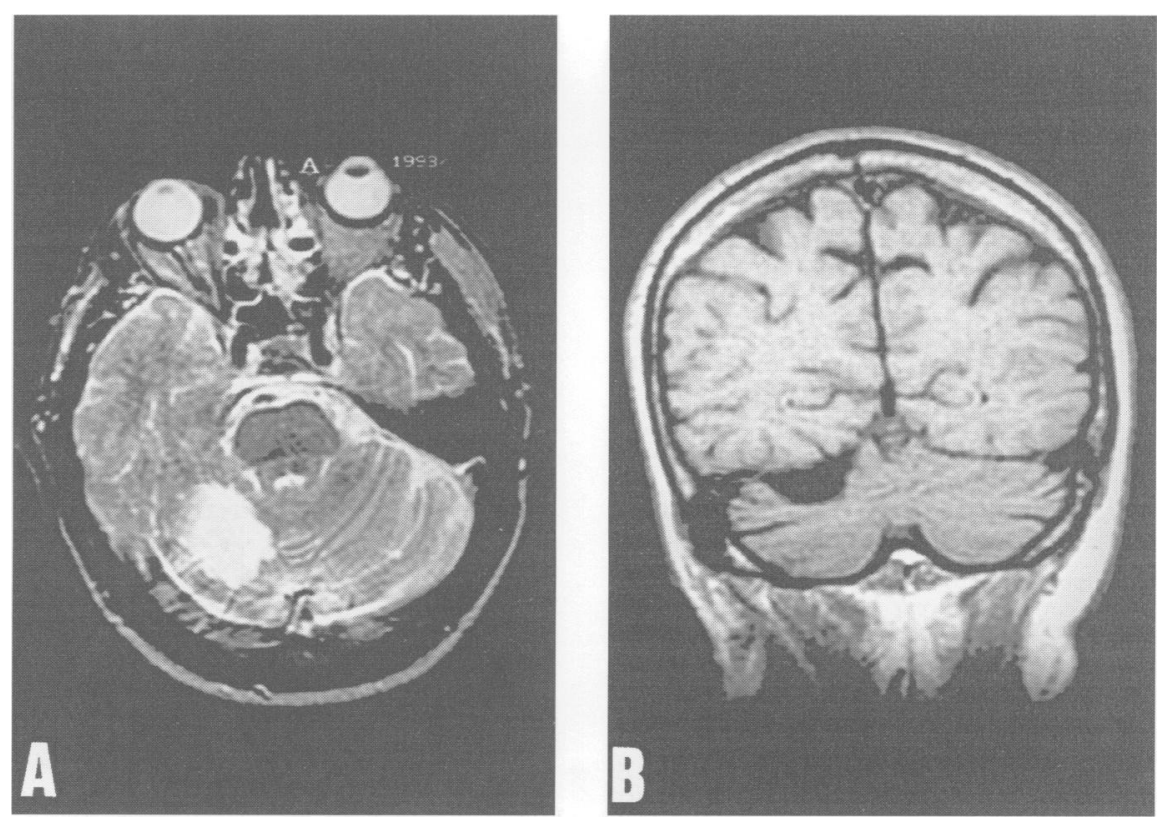

(A) Axial MRI T2 weighted scan and (B) Coronal MRI T1 weighted scan showing an infarct in the superior portion of the right paravermal zone (medial branch of the superior cerebellar artery).
The few reports agree that the paravermal zone of the rostral cerebellum is the most frequent site of damage in patients with dysarthria and focal cerebellar disorders. ${ }^{1-3}$ However, it is not clear if there is a cerebellar hemispheric dominance. In fact, topographical study of the cerebellar speech centre is difficult because most speech disorders in cerebellar lesions result from involvement of brain stem or occipitotemporal structures.

We report details of a patient with a single infarct in the right paravermal zone who presented with an isolated dysarthria.

The patient was a 78 year old right anded man (Edinburgh handedness inventory: 16) with an abrupt onset of dysarthria without other symptoms.

On admission he was alert and oriented. There was severe dysarthria, consisting of slurred articulation and exaggerated consonants. The rest of the neurological examination was normal, without gait disturbance, disequilibrium or limb ataxia, or dysphagia. His gag reflex was normal. Initial CT was normal. Brain MRI showed an ischaemic lesion located in the paravermal zone of the right rostral cerebellar hemisphere, in the territory of the medial branch of the superior cerebellar artery (figure) and recovered completely from the speech disorder within 10 days.

The patient presented with a pure cerebellar dysarthria after a right superior cerebellar artery infarction involving the right rostral cerebellar hemisphere. This area corresponds with that delineated by previous studies $^{12}$ but in the opposite cerebellar hemisphere. Thus the present case is the first report of isolated dysarthria caused by a right paravermal infarct in a right handed patient.

Dysarthria is common in superior cerebellar artery infarcts, being a characteristic sign of involvement of the medial branch of the superior cerebellar artery. ${ }^{134}$ In fact, dysarthria may be the only distinguishing clinical feature between lateral posterior inferior cerebellar artery infarcts and superior cerebellar artery infarcts. ${ }^{4}$ However,
The patient had a good clinical outcome, 
dysarthria is usually associated with other symptoms, such as vomiting, limb ataxia, or axial lateropulsion.

In posterior inferior cerebellar artery and anterior inferior cerebellar artery territory infarcts dysarthria is an unusual symptom. ${ }^{4}$ There were no detailed phonetic descriptions in the cases reported, and brain stem signs were present as well. So, it might be suspected that his speech impairment resulted from an involvement of brain stem structures.

Lechtenberg and Gilman ${ }^{2}$ first noted that dysarthria developed most often after damage extending into the superior paravermal segments of the left cerebellar hemisphere. Amarenco et $a l^{3}$ confirmed this finding in a patient with an isolated cerebellar dysarthria after a small infarction in the left paravermal zone; but they considered a minute mirror image lesion to be of no importance, the patient's hand dominance was not reported, and MRI was not performed. Nevertheless, both studies strongly implicated the left cerebellar hemisphere in the development of dysarthria. Ackermann et al ${ }^{1}$ did not corroborate the notion of an exclusive left sided paravermal cerebellar speech cortex; three of the four dysarthric patients in their study had unilateral right sided ischaemia due to occlusion of the superior cerebellar artery; all of them were right handed. The fourth had a bilateral cerebellar infarction.

More recently, increasing evidence suggests a role for the cerebellum in cognitive functions such as language. Silveri et $a l^{5}$ reported a patient with agrammatical speech after a posterior inferior cerebellar artery infarction, suggesting a right cerebellar hemispheric dominance for language function. It is not clear if that hemispheric dominance exists for speech function.

The well delineated lesion in our patient agrees with the hypothesis that the right cerebellar hemisphere is the most likely site for cerebellar speech function as cerebrocerebellar connections are predominantly contralateral and the left cerebral hemisphere is dominant for speech function in right handed people. However, it may also be possible that the site of the infarct (the paravermal zone) is more important than the side.

In conclusion, as well as describing an exceedingly uncommon clinical presentation of a superior cerebellar artery infarction, our report suggests the superior right paravermal zone as the possible site of the cerebellar speech centre, and supports the hypothesis of functional interrelation between supratentorial structures and cerebellum in functional domains outside the motor system. A GIRONELL Department of Neurology, Hospital de la Santa Creu i Sant Pau, A ARBOIX
Department of Neurology, Hospical CenA TTí JL MARTI-VILALTA Department of Neurology, Hospital de la Santa Creu i Sant Pau, Autonomous University of Barcelona, Barcelona, Catalonia, Spain

Correspondence to: Dr A Gironell.

1 Ackermann H, Vogel M, Petersen D, Poremba $M$. Speech deficits in ischaemic cerebellar lesions. $\mathcal{F}$ Neurol 1992;239:223-7.

2 Lechtenberg R, Gilman S. Speech disorders in cerebellar disease. Ann Neurol 1978;3: 285-90.

3 Amarenco P, Chevrie-Muller C, Roullet E, Bousser MG. Paravermal infarct and isolated cerebellar dysarthria. Ann Neurol 1991;30: 211-3.

4 Barth A, Bogousslavsky J, Regli F. The clinical and topographic spectrum of cerebellar infarcts: a clinical-magnetic resonance imag ing correlation study. Ann Neurol 1993;33: 451-6.

5 Silveri MC, Leggio MG, Molinari M. The cerebellum contributes to linguistic production: a case of agrammatic speech following a right cerebellar lesion. Neurology 1994;44: 2047-50.

\section{Bilateral internal carotid artery agene-} sis

Bilateral internal carotid artery agenesis is extremely rare. Agenesis of the internal carotid artery may cause cerebrovascular diseases such as subarachnoid haemorrhage or cerebral ischaemia. We describe a patient with bilateral internal carotid artery agenesis manifesting as intracerebellar haemorrhage.

A 57 year old woman suddenly developed vertigo and vomiting with severe headache and was brought to our hospital by ambulance on 19 November 1993. She was alert with slight ataxia on the right side. Brain CT showed a haematoma $2 \mathrm{~cm}$ in diameter in

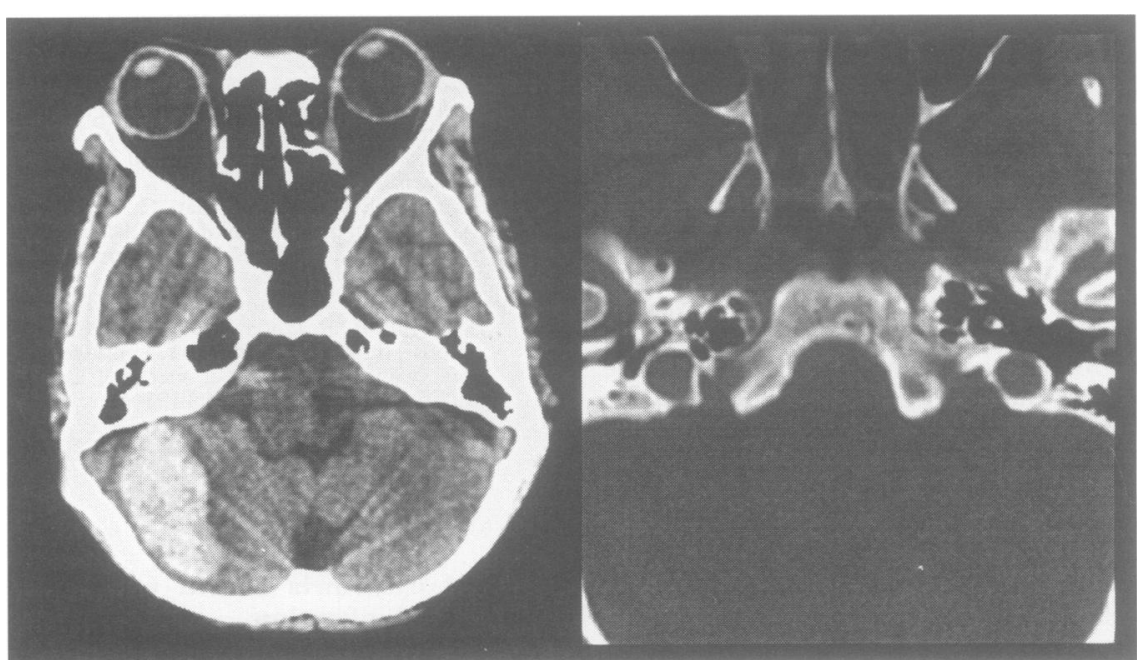

Figure 1 Left: CT showing a haematoma in the subcortical region of the right cerebellar hemisphere. Right: High resolution skull base CT showing complete absence of the carotid canals on both sides.

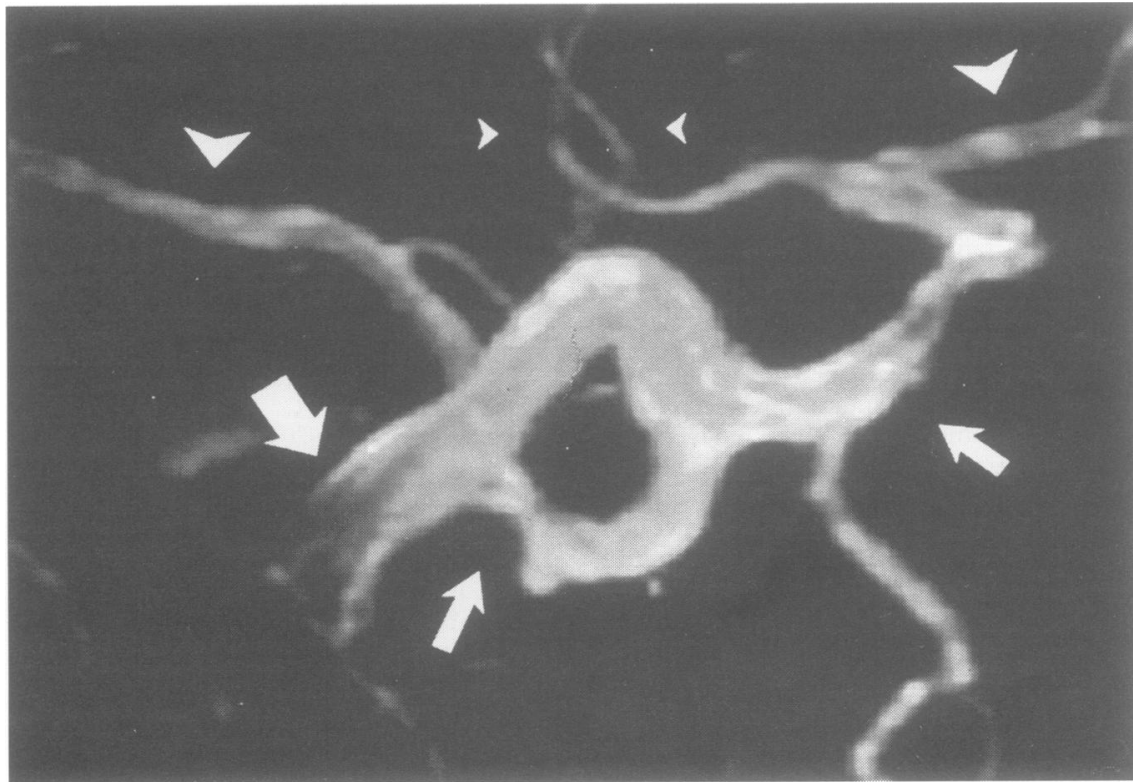

Figure 2 MR angiogram (base view) showing abnormal dilatation of the basilar artery (large arrow) and absence of the bilateral ICAs. Both anterior cerebral arteries (small arrow heads) and middle cerebral arteries (large arrow heads) were filled from the enlarged posterior communicating arteries (small arrows). the subcortical region of the right cerebellar hemisphere. Brain MRI showed no vascular malformation near the haematoma, but an angiogram (base view) showed abnorabsence of the internal carotid arteries. Both anterior cerebral arteries and middle cerebral arteries were filled from the enlarged posterior communicating arteries. Transfemoral cerebral angiography showed no bifurcation of either common carotid artery arteries. The of bilateral internal carotid plied through the middle meningeal artery. Left vertebral angiography showed an enlarged and tortuous basilar artery. A high resolution CT of the skull base showed complete absence of the carotid canals on both des, indicating

The patient gradually improved with conervative medical treatment and was dison 13 December with no home of heart failure on 30 January, 1994. Congenital internal carotid artery agenesis 